\title{
Modeling of the Cellular Communication System Coverage Area Taking into Account the External Details of the Terrain
}

\author{
Yevhen Gabrousenko \\ FAET/NAU/ Ljubomyra Guzara,1, Kyiv, Ukraine, 03058 \\ E-mail: gab58@meta.ua
}

\section{Anatolii Taranenko}

FAET/NAU/ Ljubomyra Guzara,1, Kyiv, Ukraine, 03058

E-mail: agt705@nau.edu.ua

\author{
Felix Yanovsky \\ FAET/NAU/ Ljubomyra Guzara,1, Kyiv, Ukraine, 03058 \\ E-mail: yanovsky@nau.edu.ua \\ Serhii Zavhorodnii \\ FAET/NAU/ Ljubomyra Guzara,1, Kyiv, Ukraine, 03058 \\ E-mail: zavgorodniys67@ukr.net
}

Received: 21 March 2021; Accepted: 17 May 2021; Published: 08 August 2021

\begin{abstract}
This paper proposes a new approach to estimating the contour of the coverage zone for a cellular communications base station that takes into account meaningful reflecting objects located out of the considering zone. Based on this approach, the procedure for modeling and designing the cellular system coverage area. Unlike known methods, the developed procedure considers the influence of electromagnetic wave reflection from external details of the relief, in particular essential reflecting objects located outside the considered cell. The effect of the external objects on the formation of the coverage area resulting contour is considered analytically, numerically and experimentally. The proposed solution leads to more accurate designing of the coverage area for each cell. This creates the opportunity for further development of designing techniques to more effective engineering solutions at developing and applying cellular communication systems in real situations and at various scenarios.
\end{abstract}

Index Terms: Coverage area, frequency planning, interference, radio waves, radio-electronic means, reflection, territorial planning.

\section{Introduction}

In the modern world, we are faced with the rapid and constant development of cellular communications. The need to increase the speed of information exchange and application of higher radio frequencies in the urban environment led to the fact that the phenomena of electromagnetic wave propagation are becoming more complicated under the real conditions. Predicting radio wave propagation for designing coverage areas is one of the most difficult problems in modern cellular communications. The increasing requirements are in conflict with the traditionally used simplified technologies for calculating coverage areas. This is a manifestation of the general contradiction between the needs of mankind and used opportunities for solving scientific and engineering problems.

At the same time, the shortage of the radio-frequency resource becomes more critical, and the problems of electromagnetic compatibility of radio-electronic means are becoming more complicated. Therefore, the quality of forecasting the propagation of radio waves for the design of coverage areas is becoming a priority.

Modern methods of designing the coverage areas of cellular communication systems are based on the classical theory of electromagnetic wave propagation. This approach allows qualitatively predict propagation losses of the meter and longer waves. For such frequency bands, the influence of the details of urban development and individual fragments of the terrain is practically not affected or is not critical. However, with the transition of cellular communication systems to the decimeter, centimeter, and shorter ranges, the manifestation of the terrain details has become very significant. It is important to notice that objects influencing wave propagation may be located not only inside of a 
'circle' of the zone (cell) but also outside of the particular cell.

Known techniques for predicting the propagation of radio waves and designing coverage areas do not take into account the presence of external features of the terrain as the objects which affect the behavior of the wave. We would like to fill this gap.

Let us clarify the definition first. We name details of the terrain (with all buildings and constructions) that are located outside the contour of the desired coverage area as external relief details. The presence of such external relief details can lead to additional reflections and interference of electromagnetic waves within the coverage area that can affect the resulting coverage. For example, if a high-rise building is located $100 \mathrm{~m}$ from the desired coverage area contour of $1 \mathrm{~km}$ radius, the electromagnetic wave reflected from it can be in phase or antiphase with the basic wave within the coverage area. As a result, the physical boundaries of the coverage area can change significantly. It is important to take it into account when designing the network. However, as it follows from the analysis done in the section 2, the known methods of designing coverage areas [1...9] do not take into account this phenomenon. In this paper, we make an attempt to fill this gap by proposing the solution. The originality of our solution is the analysis of the influence of external relief details on the formation of the coverage area resulting in boundaries. This should make it possible to more accurately predict the propagation of radio waves and provide the design of coverage areas of cellular communication systems that in turn will lead to reducing the probability of traffic loss, as well as the decreasing likelihood of mutual interference between neighboring cells. In practice, this allows to better solve the issues of territorial planning of cellular communications and ensuring electromagnetic compatibility.

\section{Analysis of Existing Research Results}

The article [1] examines the features of empirical models that are often used in the practice of planning wireless access networks. Based on the SUI, COST 231-Hata, Macro and, Ericsson models received signal levels are predicted for various types of urbanized environments. The $3.5 \mathrm{GHz}$ RF power measurements are comparable to those predicted using these propagation models. However, in the procedures for predicting the propagation of radio waves, the authors of this study do not take into account the features of the terrain and its external details.

The authors of the article [2] proposed a model for the propagation of radio waves on two different radio paths characterized by different types of obstacles. The results of the field strength measurements were analyzed. The analysis shows a significant discrepancy between the measured path loss and the values calculated from known statistical models. The results of approbation of the model developed by the authors confirm that for each terrain relief a certain model of radio wave propagation is required, which will allow taking into account the relief details. Such details can be large buildings and structures, as well as mountains and hills both within the coverage area and beyond its contour.

The article [3] proposes a model of a telecommunication network, which includes parts responsible for organizing access, as well as distribution and recovery segments. A number of analytical expressions are given that allow determining the number of active access equipment at all levels of the network and places for its installation based on information about the area, development, etc. But despite the fact that the authors take into account the difficult terrain, only the average radius of the circular coverage area is used in the calculations.

In the article [4] an algorithm for calculating the service areas of a cluster of base stations of cellular communication systems is developed for a given distribution of subscribers in the territory. The algorithm is based on the description of a cellular communication cluster using a system of linear equations that take into account the distribution of the load and the distance between stations. The base station load is determined as a function of the number of subscribers in the coverage area. However, despite the complex topography of urban development and the resulting possible diffraction and interference phenomena, the authors of this article assume that the coverage areas are circular.

Authors [5] directed their efforts to solve a problem associated with one of the widespread low-power wide area networks. The paper [5] presents two main clustering algorithms developed for that. The static algorithm takes into account the distribution of base stations and gateways over the network in such a way as to achieve the best coverage and maximum system reliability. The dynamic algorithm takes into account the clustering of distributed devices associated with each base station to achieve higher energy efficiency. In the process of solving these issues, the authors assume that the outer contour of the cell is circular. However, in a real environment of radio wave propagation, the shape of the cell can differ significantly from the circle.

In studies on this problem [6-8] improved methods of designing the coverage area are proposed, however, without taking into account the phenomena of reflections from external details and interference within their boundaries.

The authors of the article [9] present requirements of M\&S software for coverage analysis of the navigation system, and proposes an M\&S software design scheme on the basis of the requirements. The M\&S software is implemented, and coverage analysis is performed to verify the validity of the proposed design scheme. This study also includes the solution of conjugation of coverage areas. However, a detailed analysis of reflections from external relief details to take it into account when conjugating coverage areas was not performed in [9].

The analysis of the known research results shows that when designing and optimizing coverage areas, the influence of obstacles outside the coverage area contour is not taken into account in the known publications. However, the 
significant size of such obstacles can cause the reflection of electromagnetic waves and subsequent interference, which affects the formation of the coverage area.

Therefore, the purpose of our research is to develop a model of electromagnetic wave propagation for designing a coverage area that takes into account the presence of large relief details (buildings, structures, mountains, constructions) located outside the contour of the coverage area and causing reflection and interference phenomena within the contour. Such problem formulation did not appear in known publications.

\section{Problem Statement}

Let us look at an example of a coverage area model for a typical urban area as shown in Fig. 1, where 1 is the base station; 2 - measuring system; 3, 4-a fragment of a street; 5, 6 - buildings and structures within the contour of the coverage area. It is obvious that the propagation of radio waves in such conditions is accompanied by reflection and subsequent multi-beam interference. This leads to the formation of interference zones of maxima and minima in this area of the terrain.

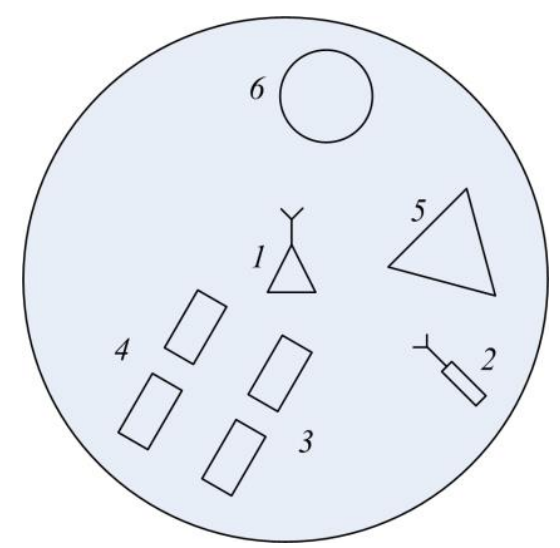

Fig.1. The scenario of coverage area modeling without accounting for external details of the terrain.

In addition, buildings and structures cause the phenomenon of diffraction and the corresponding attenuation of the radio wave. As a result, the shape of the contour of the coverage area in fact will differ significantly from the circle shown in Fig. 1. We have discussed this situation in [10]. Here we discuss new studies on how the reflection of radio waves from obstacles located outside the contour of the coverage area affects the result of this area formation. Let us take a look at Fig. 2, where near the coverage area contour, shown by a circle in accordance with Fig. 1, the high-rise buildings 7 and 8 are located. This combination of terrain fragments is typical for a medium or large city.

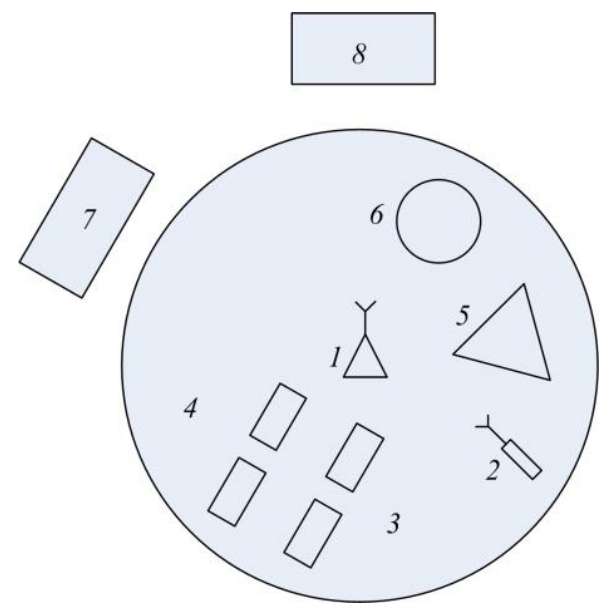

Fig.2. The scenario of coverage area modeling taking into account the external details of the terrain.

Buildings 7 and 8 cause the reflection of radio waves with certain amplitudes and phases towards the coverage area itself. At relatively short distances to these buildings, the amplitudes of the reflected waves will be comparable to the amplitudes of the direct waves propagating from the base station within the contour of the coverage area. In this case, radio waves reflected from high-rise buildings outside the contour of the coverage area can significantly affect the contour formation. This phenomenon was not taken into account in the results [10].

Known models of interference propagation [2...9] can be generally applied to analyze the influence of radio waves 
reflected from obstacles outside the contour of the coverage area on the result of this area formation. However, these models do not take into account the phenomenon of wave interference within the coverage area, when waves are reflected from external details of the relief. As a first approximation, based on the classical theory of electrodynamics we propose to take the external relief details as Huygens elements with a hemispherical radiation pattern. The initial complex wave amplitude of such elements is obtained by calculation as for a conventional reflected wave. Then, taking into account the distances to the points of observation (reception) and the difference in the path of direct and reflected waves, we will make an approximate calculation of the result of interference within the coverage area.

\section{Problem Solution}

The power of the base station (repeater) is known during the coverage design process. By its value $P_{\Sigma}$, one can calculate the amplitude of the electrical component, which is determined by the known ideal radio transmission formula [11].

$$
\dot{E}_{m}=E_{m 0} \cdot \dot{V}=\frac{\sqrt{60 P_{\Sigma} D}}{r} \dot{V}
$$

where $r$ - the distance from the source, $D$ - the directional factor of the transmitting antenna, $\dot{V}=\frac{\dot{E}_{m}}{\dot{E}_{m 0}}-$ is the attenuation factor, which is defined as the ratio of the field strength $\dot{E}_{m}$ at a distance $r$ from the transmitting antenna in real conditions to the field strength $E_{m 0}$ in free unlimited space.

Consider the phenomenon of interference using Fig. 3.

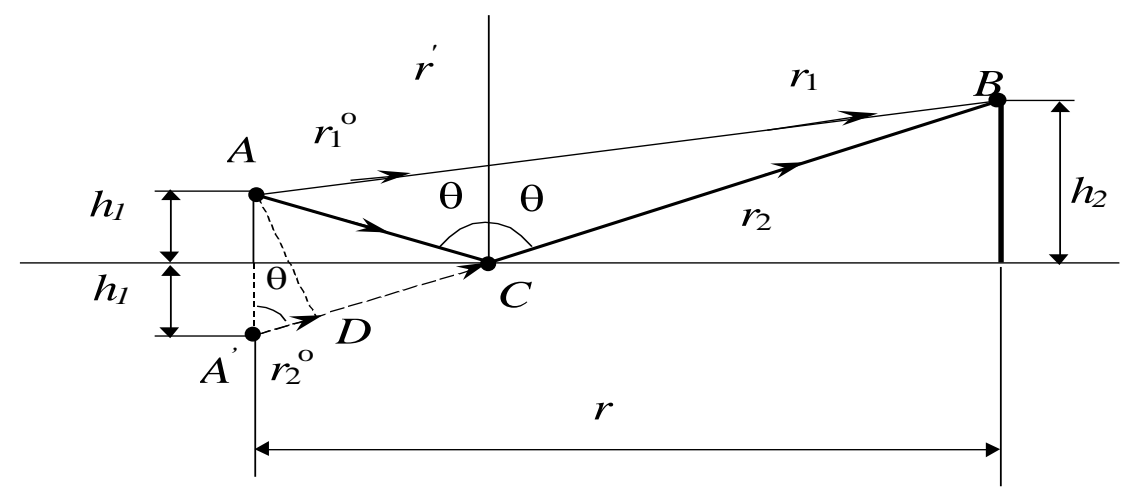

Fig.3. The simplest model of the interference phenomenon: two-path propagation case.

Taking into account multipath propagation, the resulting value of the amplitude of the field strength generated by source $A$ at point $B$, can be determined as the vector sum of the direct $\dot{\bar{E}}_{m 1}$ and reflected $\dot{\bar{E}}_{m 2}$ waves:

$$
\stackrel{\underline{\underline{E}}}{m}=\stackrel{\underline{E}}{m 1}+\stackrel{\underline{\underline{E}}}{m 2}
$$

In formula (2)

$$
\stackrel{\bar{E}}{m 1}=\bar{r}_{1}^{0} E_{m 1} e^{-i \beta r_{1}} ; \dot{\bar{E}}_{m 2}=\bar{r}_{2}^{0} E_{m_{2}} R e^{-i\left(\beta r_{2}-\psi_{r}\right)}
$$

where $\bar{r}_{1}^{0}, \bar{r}_{2}^{0}$ - unit vectors directed from points $A$ and $A$ ' to point $B$ respectively; $r_{1}$ and $r_{2}$ - distance to point $B ; R$ and $\psi-$ modulus and phase of the complex reflection coefficient from an obstacle or from the earth's surface; $\beta=\frac{2 \pi}{\lambda}-$ wave phase factor.

When calculating value $\overline{\bar{E}}_{m}(2)$, we make the following assumptions:

- distance $r$ from the source to the observation point is such that the sum of the antenna heights $h_{1}+h_{2}<r$, then the beams $A B$ and $A ' B$ (Fig. 3) are approximately parallel;

- when calculating the amplitude of the resulting wave at point $B$ we assume that $r_{1}=r_{2}=r$, and when calculating the phase shifts between the direct and reflected waves at this point, we take into account the path difference between the direct and reflected waves:

$$
A^{\prime} D=r_{2}-r_{1}=\Delta r=2 h_{1} \cos \theta .
$$


These assumptions are quite acceptable for studying the effect of wave reflection from obstacles located outside the contour of the coverage area.

Further, we substitute the values (3) and (4) into formula (2) and obtain the formula:

$$
\dot{E}_{m}=E_{m 0}\left\lfloor e^{-i \beta r_{1}}+R e^{-i\left(\beta r_{2}-\psi_{R}\right)}\right\rfloor \approx E_{m 0} e^{-i \beta r_{1}}\left\lfloor 1+R e^{-i\left(\beta r_{2}-\psi_{R}\right)}\right\rfloor=\dot{E}_{m 0}\left\lfloor 1+R e^{-i\left(\beta \Delta r-\psi_{R}\right)}\right\rfloor .
$$

The value in square brackets is the attenuation factor $\dot{V}=V \cdot e^{i \psi_{v}}$. Its modulus $V$ and phase $\psi_{v}$ are determined by the following formulas:

$$
\begin{gathered}
V=\sqrt{1+R^{2}+2 R \cos \left(\beta \Delta r-\psi_{R}\right)} \\
\psi_{v}=-\operatorname{arctg} \frac{R \sin \left(\beta \Delta r-\psi_{R}\right)}{1+2 R \cos \left(\beta \Delta r-\psi_{R}\right)}
\end{gathered}
$$

where $R$ and $\psi_{R}$ - are the modulus and phase of the reflection coefficient.

Then, on the base of formulas (2), (6) и (7), the modulus of the electric field strength can be written in the following form

$$
E_{m}=E_{m 0} \frac{\sqrt{60 P_{\Sigma} D}}{r} \sqrt{1+R^{2}+2 R \cos \left(\beta \Delta r-\psi_{R}\right)}
$$

The resulting expression indicates a periodic change in the attenuation factor depending on the difference in wave paths $\Delta r$, which corresponds to the appearance of interference maxima and minima in space.

To calculate the distance of a stable connection $r$ at known radiation power $P_{\Sigma}$ and directional action coefficient $D$, and also taking the amplitude of the intensity at the receiving point $E_{m}$ equal to the receiver sensitivity, we write formula (8) in the form

$$
r=E_{m 0} \frac{\sqrt{60 P_{\Sigma} D}}{E_{m}} \sqrt{1+R^{2}+2 R \cos \left(\beta \Delta r-\psi_{R}\right)} .
$$

The influence of electromagnetic waves reflected from external features of the relief on the formation of the coverage area can be significant at small distances from the contour of the coverage area to these details. Therefore, to take this influence into account, we suggest the following procedure of designing a coverage area. The procedure consists of 7 steps and some of them are described with more detailed sub-steps.

1. In the process of designing coverage areas, it is necessary to take into account the presence of buildings, structures and other obstacles outside its contour. This requires:

- perform a breakdown of the coverage area into sectors with an angle of no more than 5 degrees;

- separately take into account the characteristic details of the relief including buildings, structures, natural formations;

- for each selected sector, calculate the distance of a stable communications using the Walfisch-Ikegami model [10] for the phenomena of diffraction and interference. In this case, the wave intensity at the receiving point is taken equal to the sensitivity of the base station receiver;

- set transmitter power value $P_{\Sigma}$ and receiver sensitivity $E_{m}$ of the corresponding base station;

- all points corresponding to the distance of stable communications for each sector, combine with a smooth curve and get a preliminary contour of the coverage area.

2. With the help of the map, determine the location and dimensions of all substantial external relief details located directly outside the boundary of the preliminary contour of the coverage area. These may include:

- buildings and structures, the heights of which are equal to or greater than the heights of relief details within the preliminary contour of the coverage area;

- buildings and structures under construction, the design heights of which are equal to or greater than the heights of the relief details within the preliminary contour of the coverage area;

- natural relief details (hills, mountains), the height gradient of which exceeds the value of the gradient of the height of the relief details within the preliminary contour of the coverage area.

3. According to the formula (1), the field strength of the base station is determined at the place of each obstacle located outside the coverage area contour at a small distance from its boundaries, more exactly at a distance which is 
not more than half radius of the coverage zone.

4. By formulas (5) ... (8), for each selected external relief detail, the resulting field strength is determined within the limits of the coverage area contour, taking into account the reflection from the external relief details. In this case, the difference in the path of the waves can be determined by any known topographic or geometric methods.

5. A fragment of the terrain, allocated for the design of the coverage area, is divided in azimuth into sectors with a step of no more than 5 degrees. The value of the base station power $P_{\Sigma}$, as well as the field strength $E_{m}$ at the receiver sensitivity level, are set, and the stable communication distance in each sector is calculated using the formula (9).

6. Based on the obtained data and taking the value of the field strength equal to the sensitivity of the base station receiver, calculate the stable communication distance in each sector using the formula (9).

7. Points corresponding to the range of stable communications in each selected sector are marked on the map of the area of the terrain. All obtained points are connected by a smooth curve and the contour of the resulting coverage area is built.

The proposed procedure is briefly illustrated in Fig. 4.

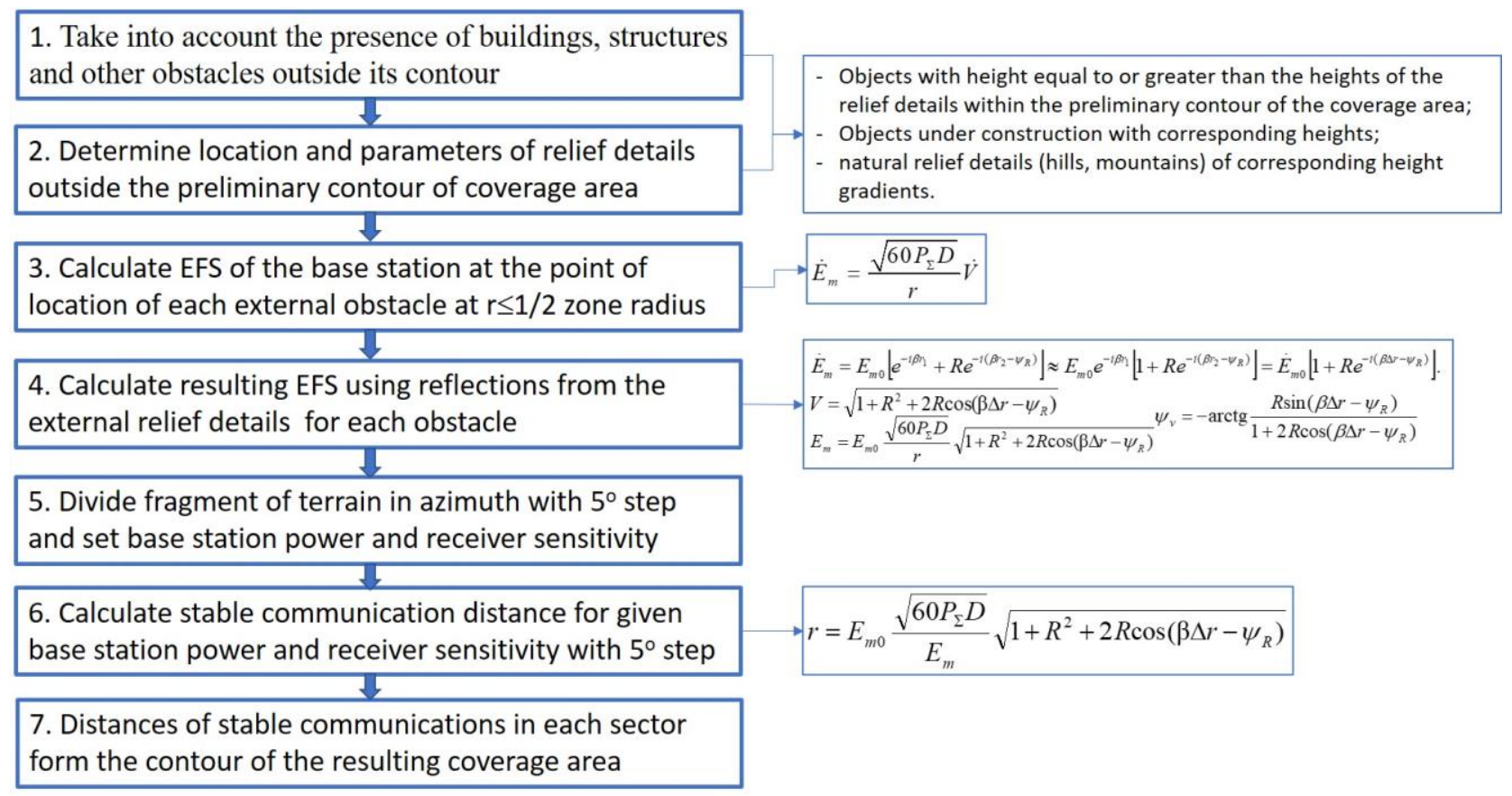

Fig.4. The developed procedure of designing a coverage area.

The simplified diagram in Fig. 4 includes also brief explanations and necessary expressions discussed above.

\section{Results of Calculations and Experimental Studies}

To carry out calculations and experimental studies according to the scenario presented in Fig. 2, the measuring kit shown in Fig. 5 was assembled. The reference designation numbers correspond to those that were previously adopted.

The laboratory setup consists of the emitter (1), a field strength detector (2), four metal objects in the form of parallelepipeds (3-4) for physical modeling of buildings, a metal pyramid (5) and a hemisphere (6) for physical modeling of structures and natural formations, metal parallelepipeds (7-8) for physical modeling of external relief details.

The experimental technique is as follows. A generator of high-frequency electromagnetic oscillations is connected to the emitter 1. The emitter 1 ensures the emission of electromagnetic waves with a uniform directional pattern in the horizontal plane. The measurement of the electromagnetic wave strength is carried out using a field detector 2 connected to the measuring receiver.

All the specified components of the laboratory setup are located on a metal circle with a diameter of $1.5 \mathrm{~m}$ with corner marks in the horizontal plane. Measurement of the dependence of the wave strength on the angle is performed by moving the field detector in the horizontal plane around the emitter. The field detector probe is oriented vertically during the measurements. 


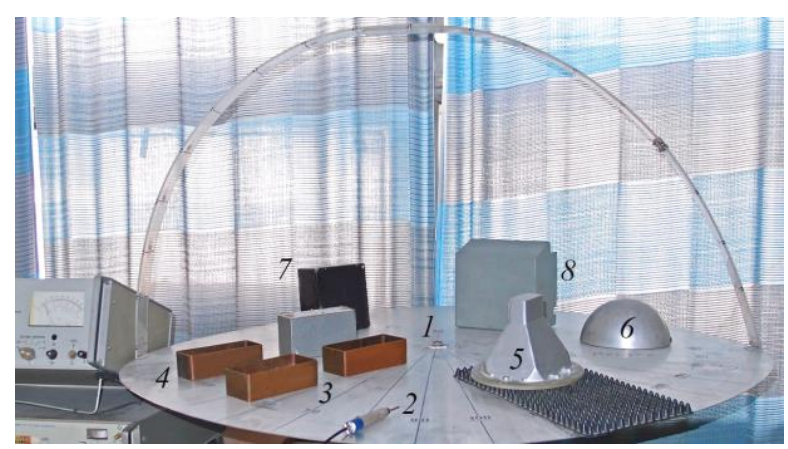

Fig.5. Laboratory setup and equipment.

In carrying out the experiment, the following values were taken, corresponding to the principles of electrodynamics modeling: the frequency of the electromagnetic wave is $12 \mathrm{GHz}$, the dimensions of the models of the relief details are $0.15 \ldots 0.35 \mathrm{~m}$. Radiation power $P_{\Sigma}=1.5 \mathrm{~mW}$; field strength at the receiving point is equal to the sensitivity threshold of the measuring receiver $E_{m}=50 \mu \mathrm{V} / \mathrm{m}$.

The given terrain relief (Fig. 2 and Fig. 4) is divided into sectors with an azimuth step of 5 degrees. Inside each sector, measurement and calculation of the value of the stable communication distance (10) with the specified initial data are made.

The results of the calculations and measurements are shown in Fig. 6, where the following designations are accepted:

1 - the contour of the coverage area calculated according to the traditional model: COST 231-Hata [1, 6];

2 - the contour of the coverage area calculated according to the proposed technique without taking into account the external details of the relief (the values of the range of stable communications as a function of the angle accounting for the reflections from the objects located inside the zone only);

3 - the contour of the coverage area calculated using the proposed technique with taking into account the external details of the relief (reflecting objects located both inside and outside the zone were taken into account);

4 - the contour of the coverage area for the values of the stable communication range determined by processing measurement data got by using the experimental laboratory setup presented in Fig. 5.

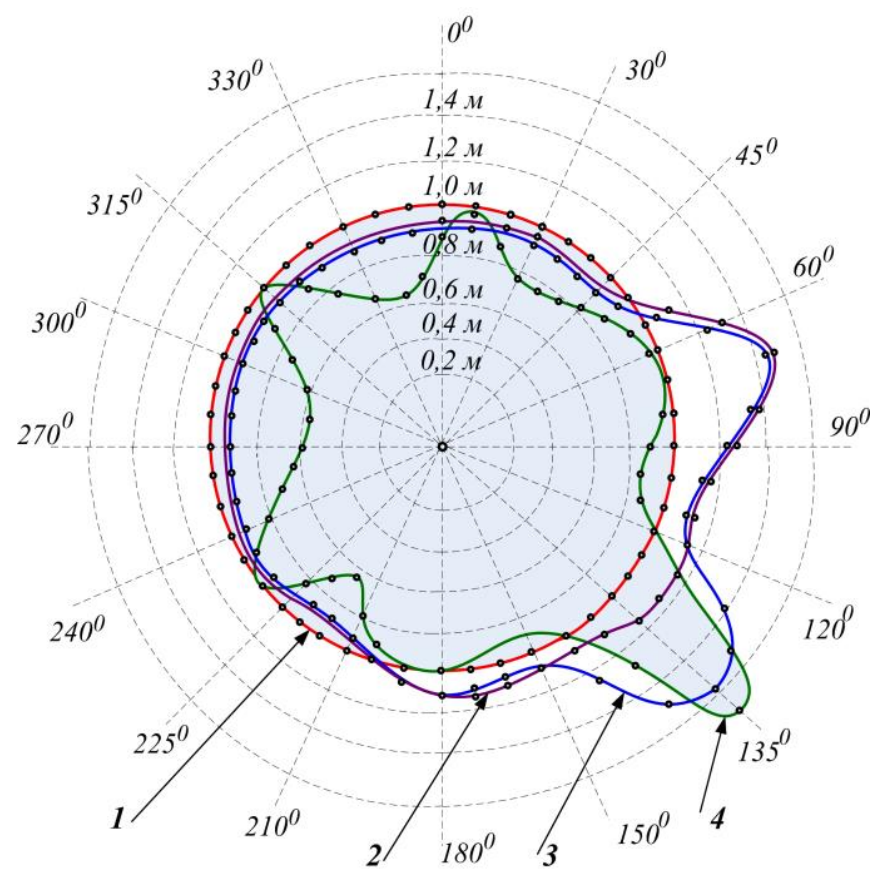

Fig.6. The results of the calculations and measurements.

The presented calculation results and measuring data processing allowed to estimate the range of stable communications at different scenarios and its dependence on the angle in the horizontal plane. In particular, the calculations based on the traditional model gave the proposed contour of the coverage area in the form of a circle, the radius of which is equal to the average distance of the stable communications and not depended on the presence and/or 
shape of the terrain features (curve 1).

The results of calculations using the proposed technique without taking into account the presence of external relief features (curve 2) is characterized by pronounced maxima and minima caused obviously by the interference of direct and reflected waves and diffraction losses of wave power.

The results of calculations using the proposed method, taking into account the influence of external relief details (curve 3), differ significantly from curve 2 due to the formation of interference maxima and minima within the contour of the coverage area caused by the additional external reflectors.

Results of measurements of the dependence of the stable communications range on the angle in the horizontal plane under the condition of external relief details presence (curve 4) has the same characteristic features as curve 3, but differs from the calculated values. The relative error is quite evident however does not exceed $24 \%$.

The indicated difference between calculations and measurements can be caused by both an imperfectness of the model and the error of measurements, in particular, due to the following reasons:

- the results of experimental measurements in practice are influenced by the geometric dimensions (effective reflecting area, like radar cross-section) of the external features of the relief, while the theoretical calculations here were carried out only taking into account the laws of geometric optics. The influence of the magnitude of the reflecting area of the relief details on the formation of the contour of the coverage area due to the complexity of the problem is supposed to be taken into account in subsequent studies;

- the process of measuring the field strength is associated with the instrumental error of the measuring instruments, the value of which is $5 \%$ for setting the generator amplitude and $2.5 \%$ for measuring the field strength with the measuring receiver.

The research results indicate a significant influence of the external details of the terrain on the formation of the coverage area resulting contour. It would be advisable to carry out such studies also for the millimeter wavelength range [12], and it is relevant for $5 \mathrm{G}$.

\section{Conclusions and Prospects for Further Research}

The problems of predicting the propagation of decimeter and centimeter electromagnetic waves, as well as designing the coverage areas of cellular communication systems, are relevant and insufficiently studied, while the quality of implementing these procedures affects directly the tasks of monitoring the frequency resource use, as well as ensuring the electromagnetic compatibility of radio-electronic equipment. For example, if the actual contour of the coverage area has pronounced minima that are not foreseen at the design stage, then this will lead to the loss of some traffic. And if the actual contour of the coverage area has pronounced maxima that were not foreseen at the design stage, then this will lead to the appearance of interference and deterioration of the quality of the base stations in neighboring cells.

Thus, the refinement of the procedures for predicting the propagation of radio waves and designing coverage areas proposed in this study will help in the practical solution of the problems of ensuring the electromagnetic compatibility of cellular communication systems. This will improve the quality of cellular services through the implementation of the following recommendations for the application of the results of this study.

The formulated brief conclusions and recommendations based on the results of this article are following.

1. Modeling the propagation of radio waves according to the proposed method, taking into account the reflection of radio waves from external details of the relief, allows to detect of the zones of the resulting minima and maxima within the coverage area and more accurately determine its contour.

2. The proposed technique for modeling coverage area differs from the known ones by taking into account the details of the relief located beyond its contour. This allows improving the quality of the procedures for coordinated use of the frequency resource.

3. The coverage area of the base station, whose parameters are previously calculated taking into account the external details of the terrain, has a shape close to the shape of the real zone. This makes it possible to more accurately place the antenna system of the base station and orient its pattern in order to reduce the mutual interference levels of different base stations that operate in the same frequency channels that have is really significant for the practice.

4. The proposed solution promotes electromagnetic compatibility of independent radio-electronic equipment by the combination of the maxima of some coverage areas with the minima of neighboring ones, and vice versa.

5. The direction of further research is the development of the proposed methodology for modeling the coverage area of cellular communication systems by determining the effective reflecting area of external details of the terrain, calculating the power of the reflected signal and determining the resulting contour of the coverage area, taking into account the interference of radio waves.

6. Similar research is reasonable to be carried out $\mathrm{mm}$ waves to cover the needs of $5 \mathrm{G}$. 
7. The next step is related to the transition from the analysis of a particular cell to design a cellular network taking into account the developed approach. This requires additional math models and further research.

\section{References}

[1] Josip Milanović, Snježana Rimac-Drlje, and Ivo Majerski, "Radio wave propagation mechanisms and empirical models for fixed wireless access systems", Technical Gazette, Vol. 17, No. 1, 2010, pp. 43-52.

[2] Jide Julius Popoola, Akinlolu Adediran Ponnle, Yekeen Olajide Olasoji, and Samson Adenle Oyetunji, "Investigation of the need for specific propagation model for specific environment based on different terrain characteristics", IIUM Engineering Journal, Vol. 19, No. 2, 2018, pp. 90-104.

[3] Kaptur V.A., and Mammadov E.M. "Opredeleniye kolichestva aktivnogo oborudovaniya dostupa i mest dlya ego ustanovki pri proektirovanii seti dostupa s uchetom osobennostei naselennogo punkta", Naukovi pratsi ONAZ im. O.S.Popova, No.1, 2015, pp. 28-35.

[4] D. Pokamestov, V. Kologrivov, G. Babur-Karatelly, and Ya. Kryukov. "Calculation of base station coverage cluster for a given subscriber distribution". Access mode http://vestnik.sibsutis.ru/uploads/1499076840_4811.pdf. Vestnik SibGUTI, No.2, 2017, pp. 26-34.

[5] Yu. T. Lyachek, M. C. A. Muthanna, S. S. S. Nasser, and M. M. A. Muthanna. "Density and distribution of LoRa basic stations in the «Smart city»". Access mode https://izv.etu.ru/assets/files/izvestiya-8-9_2020-45-52.pdf. Izvestiya SPbGETU «LETI», No.8-9, 2020, pp. 45-52.

[6] O.O.Erunkulu, A.M.Zungeru, C.K.Lebekwe, J.M.Chuma. Cellular communications coverage prediction techniques: a survey and comparison. Botswana International University of Science and Technology, IEEE Access Volume 8, 2020. Digital Object Identifier 10.1109/ACCESS.2020.3003247.

[7] Meiling Luo. Indoor radio propagation modeling for system performance prediction. Thesis for the degree of doctor of philosophy specialty "Electronics, Electrical Engineering and Automation”. National Institute of Applied Sciences Lyon, 2013. $-143 \mathrm{p}$.

[8] S. Hussain, C. Brennan. An efficient ray tracing method for propagation prediction along a mobile route in urban environments. Radio Science, Dublin City University, No 52, 2017, pp. 862-873. Access mode https://agupubs.onlinelibrary.wiley.com/doi/full/10.1002/2017RS006275

[9] Moonsuk Koo1, Young Joon Kim, Hyoungmin So, Sang Heon Oh, Seong-Cheol Kim, Dong-Hwan Hwang. Modeling \& Simulation Software Design for Coverage Analysis of Multiple Radio Positioning Integration System. Journal of Position, Navigation and Timing No.5(2), 2016, pp. 47-57.

[10] Gabrousenko Ye.I., Machalin I.A., and Taranenko A.G. "Radiowaves Propagation Model of Unmanned Aerial System Data", Proceedings of the 2015 IEEE $3^{\text {rd }}$ International Conference Actual Problems of Unmanned Air Vehicles Developments (APUAVD), Kyiv, Ukraine, October 15-17, 2015, pp. 254-257.

[11] V.O.Ivanov, E.I.Gabrusenko, and L.V.Sibruk. "Teoriya elektromagnitnogo polya", Kyiv, NAU, 2017, 336 s.

[12] P. Aruna Kumari, and I. Santi Prabha, "Signal Propagation Analysis at $28 \mathrm{GHz}$ and $73 \mathrm{GHz}$ Millimeter Wave bands for Next Generation Networks,” I.J. Image, Graphics and Signal Processing, 2019, 8, 19-27. DOI: 10.5815/ijigsp.2019.08.02.

\section{Authors' Profiles}

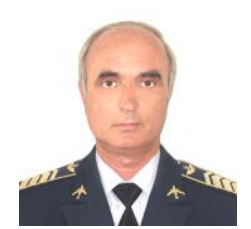

Yevhen GABROUSENKO, Candidate of Science $(\mathrm{PhD})$.

Date and place of birth: 1958, Tomsk, Russia.

Education: Kiev Institute of Civil Aviation Engineers (National Aviation University since 2000), 1985.

Affiliation and functions: $\mathrm{PhD}$ in technical sciences since 1990, associate professor of Electronics, Robotics, Monitoring and IoT Technologies Department of National aviation university since 1993.

Research interests: propagation of radio waves, electromagnetic compatibility or radioelectronic systems. Publications: more than 80 books, articles and patents.

E-mail: gab58@meta.ua

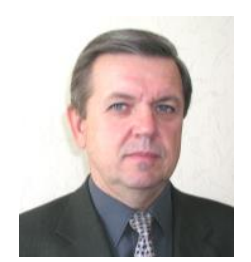

Anatolii TARANENKO, Candidate of Science $(\mathrm{PhD})$

Date and place of birth: 1956, Kiev, Ukraine.

Education: Kiev Institute of Civil Aviation Engineers (National Aviation University since 2000), 1979.

Affiliation and functions: $\mathrm{PhD}$ in technical sciences since 1989, associate professor of Telecommunications and radio-electronic systems Department of National aviation university since 1993.

Research interests: telecommunications systems, diagnostics of radio systems.

Publications: more than 60 papers.

E-mail: agt705@nau.edu.ua 


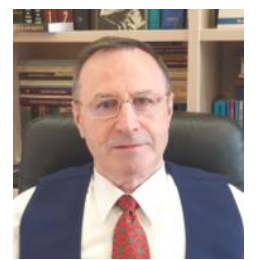

Felix YANOVSKY, Doctor of Science (DSc, PhD), IEEE Life Fellow

Date and place of birth: 1946, Kiev, Ukraine.

Education: Kiev Institute of Civil Aviation Engineers (now National Aviation University), 1968.

Affiliation and functions: Professor at Electronics, Robotics, Monitoring and IoT Technologies Department of the National Aviation University; Visiting professor at TU-Delft, The Netherlands; Kalyani Center for Technology and Innovation, Bharat Forge, Pune, India; Kenyatta University, Nairobi, Kenya; Hanyang University, Seoul city and Sunchon National University, Suncheon, Republic of Korea; Warsaw University of Technology (WUT), Poland; Beijing Institute of Metrology, China; Al-Balqa Applied University/Al-Huson University College, Jordan; Hamburg-Harburg Tech. Univ. (TUHH), Germany; PennState University, University Park, PA, USA.

IEEE Ukraine Section Chair.

Publications: 12 books, more than 480 articles, 40 invention patents.

Research interests: radar \& telecommunications, remote sensing, signal processing.

E-mail: yanovsky@nau.edu.ua

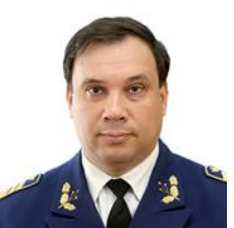

Serhii ZAVHORODNII, Candidate of Science $(\mathrm{PhD})$

Date and place of birth: 1967, Kramatorsk, Ukraine.

Education: Kiev Institute of Civil Aviation Engineers (National Aviation University since 2000), 1992.

Affiliation and functions: $\mathrm{PhD}$ in technical sciences since 2007, dean of Faculty of Air Navigation, Electronics and Telecommunications of National Aviation University since 2020.

Publications: more than 10 books, articles and patents.

Research interests: telecommunications systems, digital circuitry and modeling.

E-mail: zavgorodniys67@ukr.net

How to cite this paper: Yevhen Gabrousenko, Anatolii Taranenko, Felix Yanovsky, Serhii Zavhorodnii, "Modeling of the Cellular Communication System Coverage Area Taking into Account the External Details of the Terrain", International Journal of Computer Network and Information Security(IJCNIS), Vol.13, No.4, pp.35-44, 2021. DOI: 10.5815/ijcnis.2021.04.04 